\title{
Geomagnetic Observatories: Monitoring the Earth's Magnetic and Space Weather Environment
}

\author{
Alan W P Thomson, British Geological Survey, UK
}

\begin{abstract}
Geomagnetism research provides insight into the Earth's properties and processes, from the core out to space. For this reason continuous geomagnetic field observations have been carried out in the UK for more than 170 years. Geomagnetism also has diverse applications, in navigation, maps, even smart phone apps, and in the monitoring and prediction of space weather impacts on technology. Modern instruments, together with digital sampling, realtime data processing and product dissemination, support global space weather monitoring and modelling activities. In this review we describe the role of the UK geomagnetic observatory network in Earth and space weather science and applications.
\end{abstract}

\section{Introduction}

The Earth has many natural sources of magnetic field. A single measurement of the magnitude and direction of the field, at any given position and time, is the sum of various fields in differing proportions. The dominant source, at least in terms of measurements made at the Earth's surface, lies within the fluid, iron-rich, outer core of the planet. The outer core is a time-varying magnetohydrodynamic (MHD) dynamo. This dynamo, where the moving conducting fluid interacts with the existing magnetic field, is powered mainly by heat released by the slow solidification of the core fluid onto the inner core. Measurements at or close to the Earth's surface also record the fields of the permanently magnetised crust and of dynamic electrical currents flowing in the ionosphere and magnetosphere. To this list we can also add the fields induced in the Earth by time variations in the ionospheric and magnetospheric currents and the fields generated by ocean (water) currents and tides.

From around 1840 routine measurements of the changing magnetic field of the Earth have been made at geomagnetic observatories around the world. Together with intermittent measurements made over the centuries before that, we have built up a picture of dynamic Earth and space science processes. In the section below on 'Geomagnetic Observatories' we therefore describe the operations of the UK magnetic observatories and the international scientific and data communities in which these observatories operate.

Nowadays satellite monitoring of the geomagnetic field complements the data produced by the long term observatory network. Landmark missions, providing full vector sampling of the global field, were Magsat (1979-1980), Ørsted (1999-2013) and Champ (2000-2010). In November 2013 the European Space Agency launched the three-satellite Swarm mission. Swarm is intended to last for at least 4 years of operations and the orbital configuration will allow more precise separation of the various sources of the magnetic field. Particular 
magnetic 'highlights' from Swarm, in conjunction with the ground based data, should include finer-scale details of the Earth's structure and change, including regional-scale crustal magnetic fields and three-dimensional mantle conductivity. We will also learn more about the interaction between space weather and the geomagnetic field, including electrical current coupling between the ionosphere and magnetosphere and, from other instruments on-board, about electric fields, density and winds in the thermosphere.

The geomagnetic observatory network also provides an excellent global platform for the monitoring of space weather. In support of this network there also exist networks of geomagnetic variometers, for example SAMNET in the UK (http://spears.lancs.ac.uk/samnet/) and IMAGE in northern Europe (www.geo.fmi.fi/image/). The SuperMAG initiative (http://supermag.jhuapl.edu/) has recently brought together both observatory and international variometer networks to provide a single resource for space science and space weather studies. SuperMAG therefore provides a higher spatial density of instruments compared to the observatory network alone. However the capabilities of variometers differ from observatory instruments. For example there is limited, if any, control over the long-term absolute level of the field with a variometer: reported values can, for example, vary with air temperature. Thus variometers are not as 'broad-band' as observatory instruments and are therefore not used for determining standard geomagnetic indices of activity (see below) or for studying geomagnetic changes within the Earth. Moreover, the orientation and long-term stability of variometers is not as tightly controlled as for observatory instruments (see the discussion on INTERMAGNET below). However in terms of space weather science and services, both variometers and observatory instruments have their place.

Major space weather is accompanied by geomagnetic storms: rapid changes in the measured magnetic field caused by enhanced dynamic ionospheric and magnetospheric electrical currents. Geomagnetic data are therefore widely used for parameterising models of the nearEarth environment and for validation of those models. Rapid geomagnetic variations, through Faraday's law of electromagnetic induction, induce electric fields in the conducting Earth. These fields cause currents to flow in high voltage power systems, posing a threat to the transmission system and to the transformers within it. Rapid magnetic field changes also pose a hazard to other grounded infrastructure, such as pipeline networks and railways. In the section below on 'Geomagnetism and Space Weather' we therefore review how UK geomagnetic observatory data are used in relation to space weather monitoring and forecasting and, in particular, in the analysis of the hazard to electrical power transmission in the UK.

\section{Geomagnetic Observatories}

In the UK there are three permanent geomagnetic observatories: Lerwick in Shetland, Eskdalemuir in the Scottish Southern Uplands, and Hartland on the north Devon coast. Hartland is the most recently established observatory (1957), a successor to observatories 
located at Greenwich (1836-1926) and latterly Abinger in Surrey (1924-1957). Eskdalemuir (established 1908) is the successor to the Kew observatory (1857-1924) in London. (That relocation of observatories is necessary is a consequence of urban development and growth in man-made electromagnetic noise.). The Lerwick observatory (where magnetic observations began in 1922) is situated on land owned by the Met Office, whilst the British Geological Survey (BGS, Natural Environment Research Council) hosts the Met Office at its Eskdalemuir site. The UK observatories are part of an international network of some 160 observatories, including five other, non-UK, observatories operated by BGS, as shown in Figure 1.

The UK observatories currently operate Geomagnetic Data Acquisition Systems (GDAS), providing a robust and reliable continuous sampling of the magnetic field, with three identical systems at each site giving necessary redundancy and therefore 24/7 data availability. The vector instrument used is a FGE fluxgate magnetometer manufactured by the Danish Technical University. Data are sampled at $1 \mathrm{~Hz}$, with a resolution of $0.2 \mathrm{nT}$. Operating nearby and in parallel, a GEM Systems GSM-90 proton precession magnetometer samples the magnitude, or intensity, of the field at a 10 -second period, with $0.1 \mathrm{nT}$ resolution. GDAS timing is GPS referenced and the data loggers connect with the BGS Edinburgh office via internet, telephone and satellite links. All equipment and calibrations are regularly checked against National Accreditation of Measurement and Sampling (NAMAS) standards.

All automated measurements are referenced against regular, bi-weekly, manual measurements to provide full 'baseline' control, such that the automated data can be published as absolute values. Manual measurements of declination (or 'compass variation') and inclination (or 'dip') are made using a Bartington MAG 01H fluxgate magnetometer, attached to either a Zeiss or Wild theodolite. These measurements are used to correct the automatically sampled vector and scalar valued data to a single reference point at the theodolite location.

The standard UK observatory data product is a one-minute filtered version of the one second vector data. Filtering is according to the INTERMAGNET standard (a 91-second Gaussian filter, centred on the minute). INTERMAGNET is a consortium of observatories and operating institutes that agree and stipulate standards for worldwide magnetic observatories (www.intermagnet.org). Derived observatory data products include hourly, monthly and annual means of the one-minute data and more bespoke data products have also been developed for space weather monitoring. Both INTERMAGNET and non-INTERMAGNET data and products (including older data and survey data) are held at the various World Data Centres for Geomagnetism, e.g. in Edinburgh (www.wdc.bgs.ac.uk).

Geomagnetic variations can be summarised through a variety of magnetic activity indices, such as the observatory $K$ scale; a quasi-logarithmic measure on a scale of 0 to 9 (e.g. Mayaud, 1980, for a summary). Regional and global magnetic indices are also produced by combining various observatories' data. Indices of activity in mid-latitudes include the planetary-scale, three-hour range indices, $k p$ and $a p$, and the $a a$ index, which is simply constructed from data from near antipodal observatories at Hartland and Canberra, Australia. 
The planetary ap index is a version of the $k p$ index, but in physical units. Daily versions of indices are also produced.

As a new development, and as part of the support for the ESA Swarm 'Earth Explorer' mission, INTERMAGNET has promoted the production of Quasi-Definitive Data (QDD). What are known as the observatory definitive data may take some months, if not years, to finalise. Therefore, to maximise the value of Swarm and its data during the mission itself, a more quickly released, but reliable, ground-based data product is required. Though there are many processing details, in essence QDD provide an estimate of the absolute level of magnetic field, using a predicted baseline, either in real time or within a few weeks of measurement. In practice (e.g. Clarke et al., 2013) QDD values prove to be within +/-5 nT of the definitive observatory data with a very high reliability. This provides modellers with an appropriate level of accuracy for improving current models of the sources of the magnetic field.

Geomagnetic observatories in the UK can genuinely be regarded as general 'geophysical observation sites'. The UK observatories host instruments from a number of institutes and universities measuring atmospheric electricity, seismic activity, VLF waves and providing gravity and GPS referencing. A recent development at the UK observatories has seen the installation of geo-electric (surface electric field) monitoring equipment, to support research into the space weather hazard to the power transmission system. Because of the infrastructure at the observatories and the availability of real-time links to the BGS Edinburgh office, other institutes are actively encouraged to use these remote facilities.

\section{Geomagnetism and Space Weather}

Space weather refers to the natural variability of the Earth's space environment, which includes variability in the ionosphere and magnetosphere and, beyond that, the solar wind. As described earlier, the variability of electrical currents that flow in the ionosphere and magnetosphere is a natural hazard to technology, for example by driving unwanted and potentially damaging induced currents in electrical transmission systems on the Earth.

The Earth's magnetosphere is a complex nonlinear system, containing charged particles whose dynamics is influenced by the magnetic field, through MHD processes. Magnetospheric activity can be driven by the solar wind electric field, when the interplanetary magnetic field (IMF) has a 'southerly' direction, allowing a magnetic connection to be made on the day side of the Earth between the IMF and the Earth's own field. This connectivity extracts energy and plasma from the solar wind and transfers magnetic flux from the day side of the Earth into the night side magnetosphere. Flux transfer is balanced by return processes operating in the night side magnetosphere on different timescales.

These night side processes channel currents from the magnetosphere into the high-latitude ionosphere at about $100 \mathrm{~km}$ altitude in ovals around 10-30 degrees from each magnetic pole, 
where the associated aurora occurs. Such currents are called the auroral electrojets. Another major magnetospheric current system, in terms of activity that causes problems for groundbased technology, is the equatorial ring current circling the Earth at around 5-7 Earth radii (Re) distance. Also, the sudden compression of the magnetosphere, that marks the onset of a geomagnetic storm, can generate currents in the magnetopause ( 10Re) that drive induced currents in grids.

Despite the complexity of the system, in terms of the impact at ground level it is the time rate of change of the magnetic field, $\mathrm{d} B / \mathrm{dt}$, which induces currents in grounded infrastructure. That is, whether the source is magnetopause, ring or auroral electrojet currents, a 'sufficiently large' rate of change is needed to cause risk to any earthed system. Due to the proximity to the source, activity in the auroral electrojets often drives the largest and most rapid $\mathrm{d} B / \mathrm{dt}$. These are described as substorm events, lasting a few hours at most, and are distinguished from geomagnetic storms lasting a few days, which are associated with changes in the ring current (though substorms also occur during storms). In Figure 2 we show just how well $\mathrm{d} B / \mathrm{dt}$ acts as a proxy for measured geomagnetically induced currents (GIC) in a typical high voltage transformer. This also indicates the value of real-time monitoring of the geomagnetic field in the situation where not all transformers can be instrumented to measure GIC.

From an understanding of the geophysical structure of the UK and Ireland, as well as the bathymetry of the off-shore region, we can develop models of the induced electric field (Figure 3), which depends on the conductivity of the land and sea as well as rapid changes in the geomagnetic field (Thomson et al., 2005; Beggan et al., 2013). This geoelectric field is essentially a risk map for the power system, in that high potential differences, found typically near the coast or at major geological boundaries, will drive larger GIC. By using the geoelectric field model together with a DC network model of the power transmission system one can calculate the flow of GIC at each major substation and transformer in the system (e.g. Beggan et al., 2013). Then, with access to real-time geomagnetic data as input, such coupled models can be used to provide a 'now-cast' of conditions in the grid. Indeed a web-based monitoring and analysis tool ('MAGIC') has been developed by BGS and National Grid and is in operational use by the latter.

Routine measurement of the geoelectric field is now carried out at the UK observatories. This has validated the electric field modelling to some extent, given the clearly limited sampling of the UK mainland, but has also revealed features that are not in models. A particular example of this is shown in Figure 4. Near the coast, the ocean tidal influence is clear. This is most marked at Hartland, given the $7 \mathrm{~m}$ tidal range of the Bristol Channel. This suggests improvements need to be made to the basic geoelectric model to improve the agreement with measurements and hence improve GIC estimates.

Geomagnetic field measurements at the UK observatories find use in other areas of Earth and space science. For example, induction coil magnetometers at the observatories are used to measure long term and seasonal trends in atmospheric electricity, through detection of Schumann resonances. The geomagnetic indices that the UK observatories contribute to are widely used, for example, in thermospheric neutral density models, (e.g. Hedin, 1987), to 
characterise the atmospheric heating by the coupled ionospheric and magnetospheric current systems. Finally, geomagnetic observations and forecasts of activity help promote interest in the aurora borealis (e.g. University of Lancaster’s AuroraWatch: @aurorawatchuk; http://aurorawatch.lancs.ac.uk/ and BGS: @BGSspaceWeather; @BGSauroraAlert; http://www.geomag.bgs.ac.uk/ data_service/space_weather/alerts.html)

\section{Acknowledgements}

The author acknowledges INTERMAGNET, the World Data Centres for Geomagnetism and the global geomagnetic observatory network as sources for data. Thanks are also due to Susan Macmillan for the observatory map (Figure 1) and Gemma Kelly for preparation of the geoelectric field data and maps (Figures 3 and 4). Susan Macmillan is also thanked for discussions and comments on the manuscript. This paper is published with the permission of the Executive Director of the British Geological Survey (NERC).

\section{References}

Beggan CD, Beamish D, Richards A, Kelly GS, Thomson AWP. 2013. Prediction of extreme geomagnetically induced currents in the UK high-voltage network. Space Weather, 11: 1-13, doi:10.1002/swe.20065.

Clarke E, Baillie O, Reay SJ, Turbitt, CW. 2013. A method for the near real-time production of quasi-definitive magnetic observatory data. Earth Planets Space, 65: 1363-1374.

Hedin AE. 1987. MSIS-86 thermospheric model. J. Geophy. Res., 92, A5: 4649-4662, Mayaud, P. 1980. Derivation, meaning and use of geomagnetic indices, Geophysical Monograph, 22.

Thomson AWP, McKay AJ, Clarke E, Reay SJ. 2005. Surface electric fields and geomagnetically induced currents in the Scottish Power grid during the 30 October 2003 geomagnetic storm. Space Weather, 3: S11002, doi:10.1029/2005SW000156. 


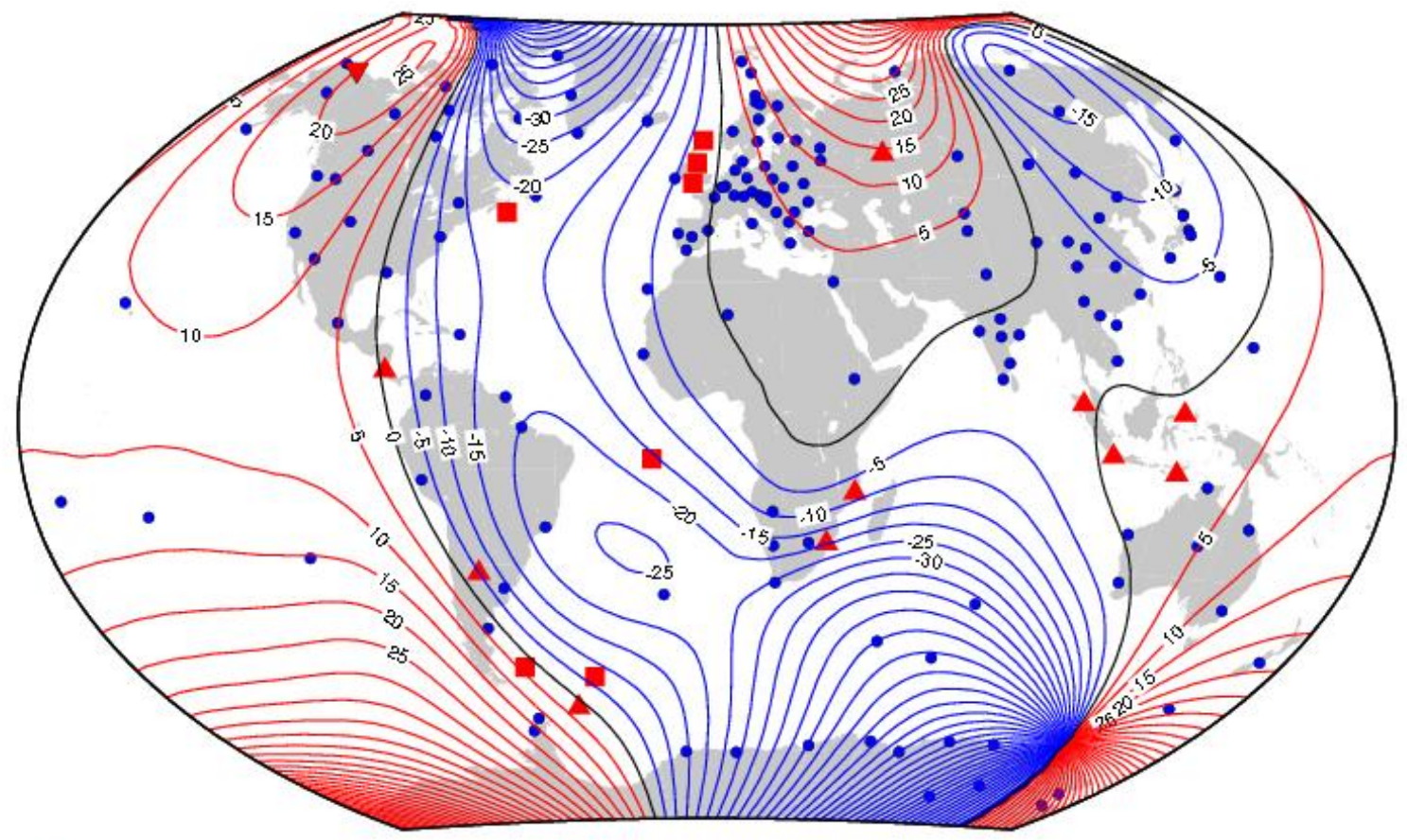

Figure 1. Worldwide distribution of geomagnetic observatories in 2013. Squares indicate observatories operated by BGS, triangles indicate observatories operating instruments supplied by BGS to partner institutes, and spots indicate locations of other observatories. The contours show the compass direction at 2013, with blue/red representing west/east of true north. Black contours indicate where true and magnetic north are aligned. 
Measured Scottish Grid GICs Compared With Eskdalemuir dX/dT for 6th-7th April Magnetic Storm

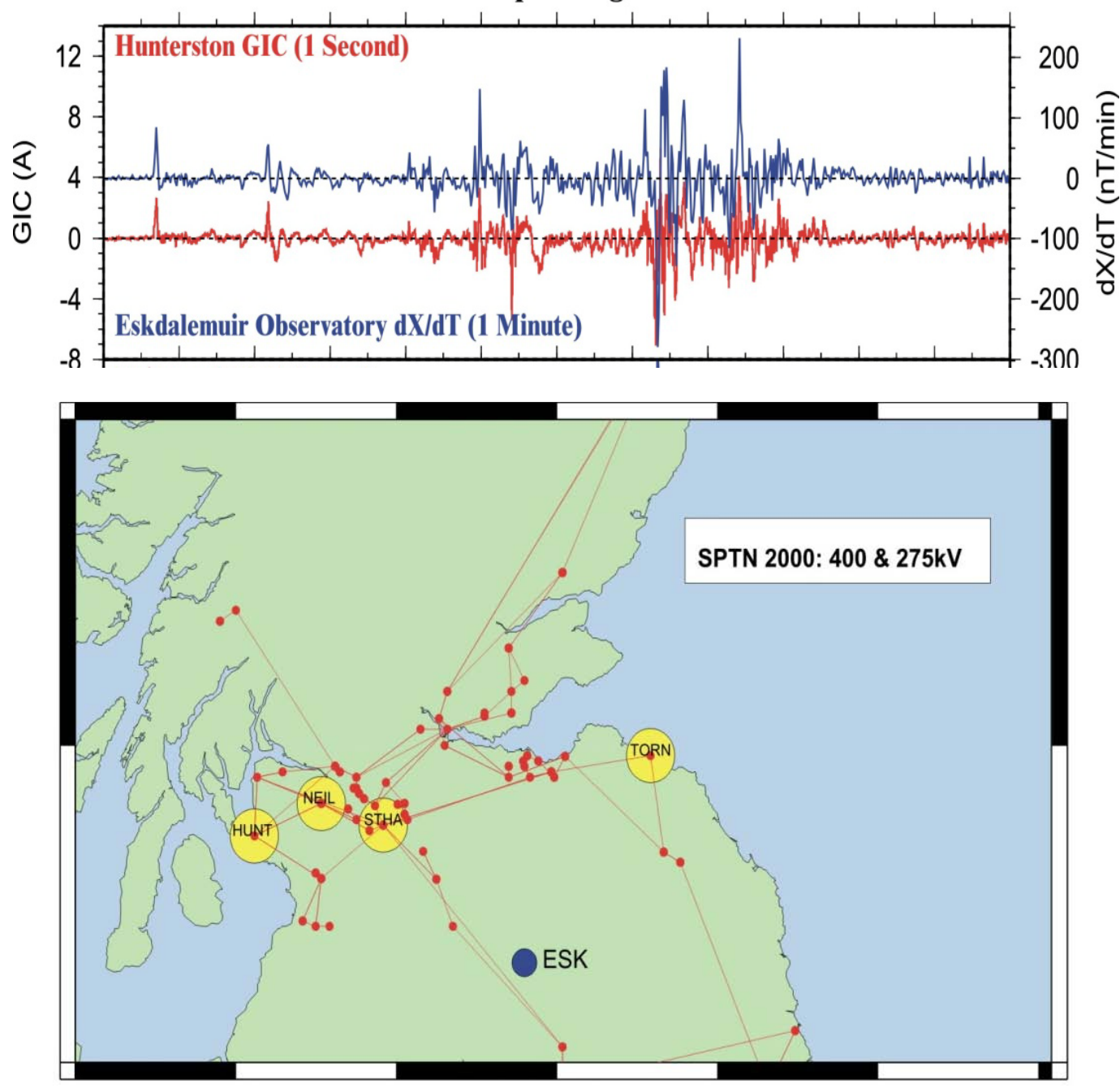

Figure 2. Top: measured GIC (red) at one site in the central Scottish transmission system and $d B / d t$ (blue, where $X=$ north pointing component) at the Eskdalemuir observatory, for a storm in April 2001. Bottom: locations of measurement sites (blue: Eskdalemuir observatory; yellow: GIC monitors) and the local high voltage transmission system (Scottish Power Transmission Network). 


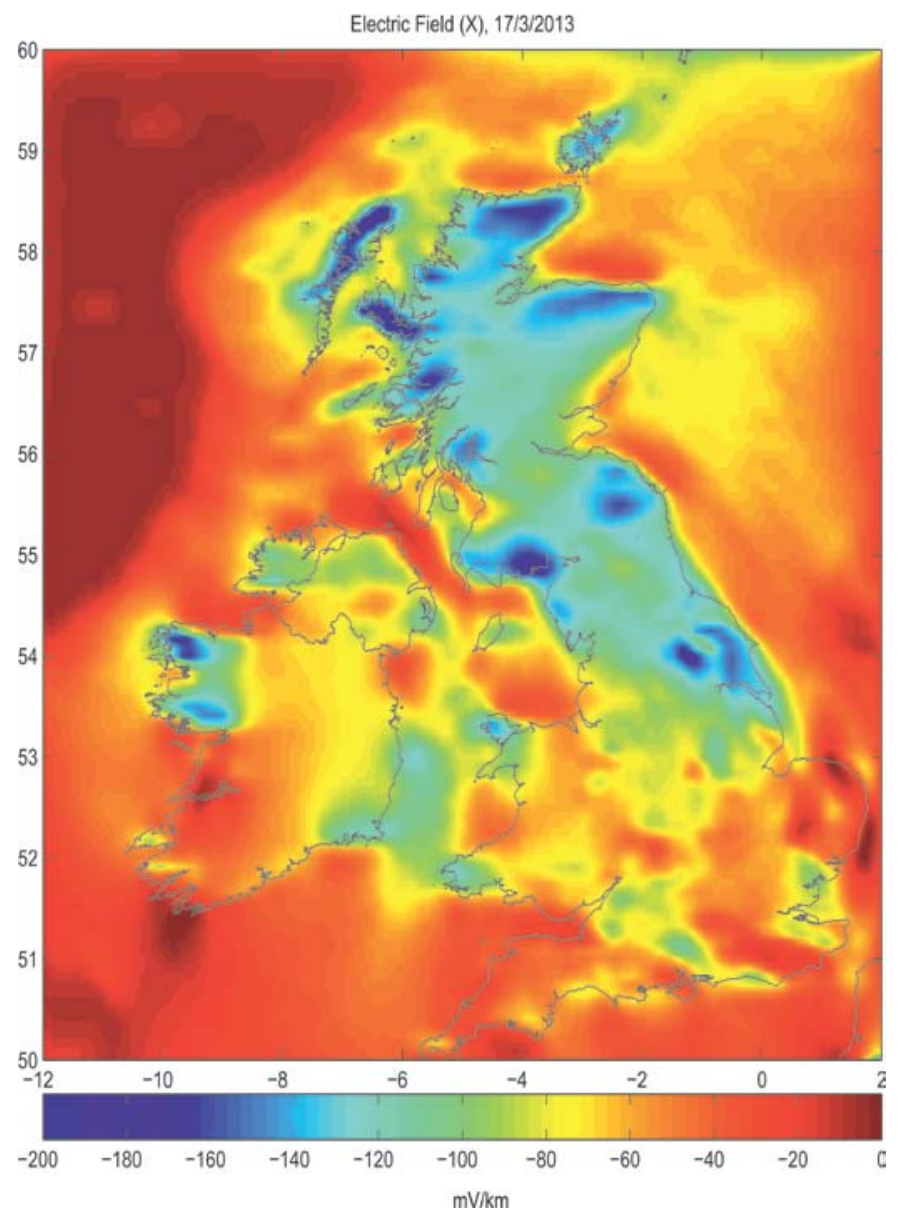

Figure 3. Map of modelled UK geoelectric field in the north direction at the time of the peak in a geomagnetic storm on $17^{\text {th }}$ March 2013. Strong coastal contrasts are evident, reflecting differing onshore and offshore conductivities. 

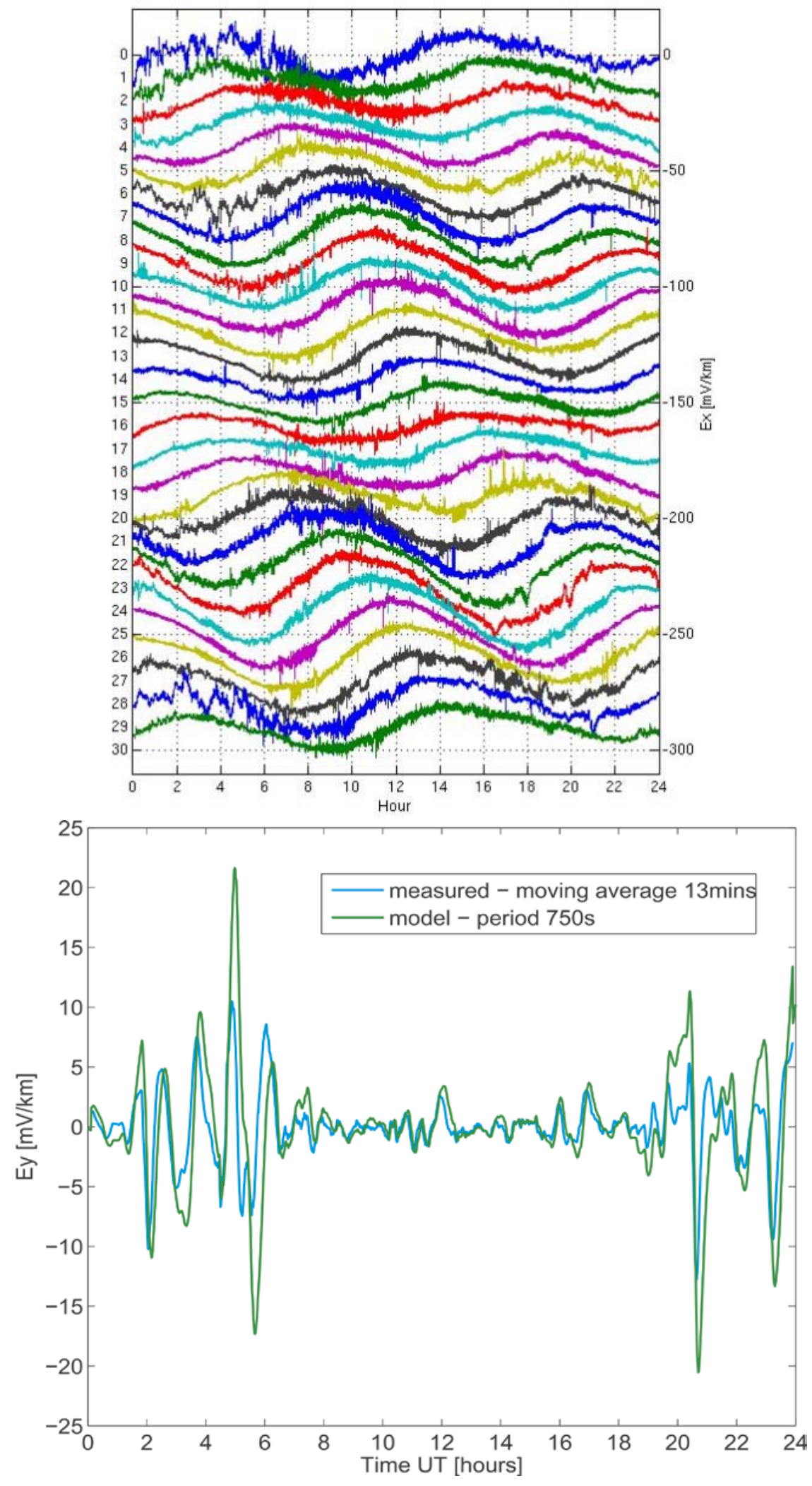

Figure 4. Measured and modelled geoelectric field at Hartland observatory in $m V / \mathrm{km}$. Top: Stack plot of 30 days in June 2013 of measured geoelectric field showing a strong ocean tidal signature. Daily plots are offset by $10 \mathrm{mV} / \mathrm{km}$ for clarity. Bottom: comparison of measured and modelled easterly geoelectric field after tidal signature is removed. Measured data are 13-minute filtered to match model characteristics. 